\title{
Long-term treatment with olanzapine increases the number of Sox2 and doublecortin expressing cells in the adult subventricular zone
}

\author{
Barbara Łasut ${ }^{1}$, Artur Pałasz ${ }^{1}$, Łukasz Filipczyk ${ }^{1}$, Oscar Arias-Carrion ${ }^{2}$, Ewa Rojczyk ${ }^{3}$, \\ Mariia Savchyna ${ }^{4}$, Katarzyna Bogus ${ }^{1}$, John J. Worthington ${ }^{5}$, Marek Krzystanek ${ }^{6}$, \\ Ryszard Wiaderkiewicz ${ }^{1}$
}

${ }^{1}$ Department of Histology, School of Medicine in Katowice, Medical University of Silesia, ul. Medyków 18, 40-752, Katowice, Poland

${ }^{2}$ Unidad de Trastornos del Movimiento y Sueño/Centro de Innovación Médica Aplicada, Hospital General Dr. Manuel Gea Gonzalez, México City, México

${ }^{3}$ Department of Descriptive and Topographic Anatomy, School of Medicine with Division of Dentistry in Zabrze, Medical University of Silesia, ul. Jordana 19, 41-808, Zabrze, Poland

${ }^{4}$ Department of Histology and Embryology, Bogomolets National Medical University, Kyiv, Ukraine

${ }^{5}$ Division of Biomedical and Life Sciences, Faculty of Health and Medicine, Lancaster University, Lancaster, LA1 4YQ, UK

${ }^{6}$ Department and Clinic of Psychiatric Rehabilitation, School of Medicine in Katowice, Medical University of Silesia, ul. Ziolowa 45/47 40-635, Katowice, Poland

Corresponding author:

Dr Artur Pałasz, Medyków Street 18, 40-752, Katowice, Poland.

Email: apalasz@sum.edu.pl; Phone: +48 322088377 
Abstract

Continuously active neurogenic regions in the adult brain are located in the subventricular zone (SVZ) of the lateral ventricles and subgranular zone (SGZ) of the hippocampal dentate gyrus. Neurogenesis is modulated by many factors such as growth factors, neurotransmitters and hormones. Neuropsychiatric drugs, especially antidepressants, mood stabilizers and antipsychotics may also affect the origin of neuronal cells. The purpose of this study was to determine the effects of chronic olanzapine treatment on adult rat neurogenesis at the level of the SVZ. The number of neuroblasts was evaluated using immunohistochemical and fluorescent detection of sex determining region Y-box 2 (Sox-2) and doublecortin (DCX) expressing cells. The results indicate that olanzapine has proneurogenic effects in the adult rat SVZ, as the mean number of SOX-2 and DCX-positive cells increased significantly, while there was a similar tendency in the SGZ. Collectively, these results suggest that long-term treatment with olanzapine may stimulate neurogenic stem cell formation in the SVZ which supports adult neurogenesis.

Key words;

adult neurogenesis, olanzapine, DCX, Sox-2, neuroleptics 


\section{INTRODUCTION}

New neurons are continuously being generated in the adult mammalian brain. This process is initiated in adult neural stem cells (NSCs), whichreside mainly in two canonical neurogenic brain regions - the subventricular zone (SVZ) of the lateral ventricles and the subgranular layer of the hippocampal dentate gyrus (SGZ) [1-3]. In vivo studies have shown that various growth factors, neurotransmitters, drugs or brain injury and degenerative neurological diseases affect the proliferation, migration and differentiation of adult NSCs[1, 2, 4-7]. The process of adult neurogenesis has been a challenge for many scientists around the world. Understanding the mechanisms and the factors regulating this phenomenon could become a basis for developing new models for the pathogenesis of neurodegenerative diseases, a growing welfare and financial burden in the ageing population.

Olanzapine, an atypical antipsychotic agent, has an affinity to various brain receptors including dopaminergic $\left(D_{1}-D_{5}\right), \alpha_{1}$-adrenergic, muscarinic cholinergic $\left(M_{1}-M_{5}\right)$, histaminergic $\left(\mathrm{H}_{1}\right)$ and serotoninergic $\left(5-\mathrm{HT}_{2 \mathrm{~A} / 2 \mathrm{C}}, 5-\mathrm{HT}_{3}, 5-\mathrm{HT}_{6}\right)[8]$. It selectively reduces the stimulatory activity of dopaminergic neurons in the mesolimbic system, but at the same time has only a minor influence on striatum neuronal pathways involved in motor functions[9]. Olanzapine reduces both negative and positive schizophrenia symptoms and shows mood stabilizing activity[10]. Some studies also suggest a possible connection between the activity of brain regions involved in emotional processes and prolonged olanzapine intake by patients with schizophrenia[10]. Indeed, olanzapine increases the activity of the limbic structures associated with the feelings of anxiety and with stress response generation[8]. The aim of our pharmacological study was to check the potential modulatory effect of olanzapine on adult neurogenesis in a rat animal model by the evaluation of NSCs and neuroblast marker

expression. The following markers have been chosen: sex determining region Y-box 2 (Sox-2), a transcription factor that enables the self-renewal of neural stem cells and doublecortin (DCX), a microtubule-associated protein selectively expressed by immature neurons. Here, we investigated the effect of the long-term (4 week) administration of olanzapine on proliferation and migration by counting Sox2 and DCX-positive cells in the adult SVZ in rats. 


\section{MATERIAL AND METHODS}

The studies were carried out on adult (2-3 months old, 180-220 g) male SpragueDawley rats from the Medical University of Silesia Experimental Centre. Animals were housed at $22^{\circ} \mathrm{C}$ with a regular $12 / 12$ hour light-dark cycle with access to standard Murigan chow and water ad libitum. All procedures were approved by the Local Bioethics Committee at the Medical University of Silesia (decision no. 36/2012) and were conducted in a manner consistent with NIH Guidelines for Care and Use of Laboratory Animals.

Two groups of animals (5 individuals each) had received respectively control vehicle or olanzapine $(10 \mathrm{mg} / \mathrm{kg} / \mathrm{day}$, dissolved in isotonic saline) by intraperitoneal injection for 4 weeks. 24 hours after the last drug administration, rats were anesthetized with isoflurane and then immediately sacrificed by decapitation. Rat brains were then excised, fixed with 4\% paraformaldehyde PBS ( $\mathrm{pH} 7,2-7,4)$, dehydrated, embedded in paraffin and finally sectioned on the microtome (Leica Microsystems, Germany) in the coronal plane for the SVZ $(0,36$ to $-0,50 \mathrm{~mm}$ from bregma) at $7 \mu \mathrm{m}$ thickness.

After blocking with $5 \%$ goat serum, sections were incubated overnight with a goat antibody against rat doublecortin (DCX) (1: 1000, Santa Cruz Pharmaceuticals) and rabbit antibody against rat Sox2 (1: 1000, Abcam). Primary antibodies were followed by biotinylated goat anti-rabbit secondary antibodies, and then an avidin-biotin-horseradish peroxidase complex (Vectastain ABC kit, Vector Labs). Finally, 3,3'-diaminobenzidine (DAB) was used to complete the visualization reaction. All sections were mounted on glass slides with amedium, dehydrated and coverslipped. Additionally, after overnight incubation with primary antibodies for Sox2, several brain sections were kept in darkness with secondary antibodies labeled with FITC (1:200, Abcam) and then, mounted on slides with the DAPI-containing medium. For calculation of DCX-positive cells, 10 slices (every tenth one) per rat for each brain region were used. All images ( 8 per slice) were captured with the Nikon Coolpix optic system and processed using Image ProPlus software (Media Cybernetics, USA). Anatomically comparable sections were analyzed and immunopositive cells were counted using ImageJ $1.43 \mathrm{u}$ software. We counted the total number of DCXpositive cells in the neurogenic zones for each rat (which was the sum of cells from 10 slices) and divided the results per length of the analyzed SVZ to obtain the density of 
immune-positive cells per one millimeter of length. Data are presented as a mean \pm standard error of the mean (SEM).

Statistical analyses were performed using Statistica (Systat Software). Mean differences between experimental groups were analyzed using non-parametric KruskallWallis test. Differences were considered statistically significant at $p \leq 0,05$.

\section{RESULTS}

The mean number of Sox-2-immunopositive cells in the SVZ of animals exposed to olanzapine was $4904 \pm 748$, whereas in controls was $2471 \pm 1504$, $p=0,043$ (Fig 1. A,B and Fig 2.). The number of DCX-immunoreactive cells in SVZ was $1656 \pm 444$ for animals treated with olanzapine and $1081 \pm 364$ for the control group, $p=0,286$ (Fig 1. C,D). Thus, the increase in neuroblast formation in the SVZ was $21.11 \%$. All relatively densely packed DCX-immunopositive neuroblasts were located beneath the wall of lateral ventricles (Fig 1 C,D). Interestingly, analyzed slices containing the hippocampus, DCX-immunoreactive cells were dispersed in the subgranular zone (SGZ) of the dentate gyrus and contrary to what we show in the SVZ, neuroblasts in the SGZ do not form any clustered forms (data now show).

\section{DISCUSSION}

There are two groups of antipychotics involved in adult neurogenesis: 1) atypical antipsychotics e.g. olanzapine, clozapine, risperidone; and 2) typical (classical) neuroleptics - mainly haloperidol and chlorpromazine. These studies show unclear data about the positive effects of olanzapine on neurogenesis [11-13]. Here, we show a clear difference in the number of neuroblasts in the SVZ after long-term treatment with olanzapine. The first studies on haloperidol effect on cell proliferation were performed on young rats (11- days old) [14]. They reported that 24 hours after drug administration (single dose of $20 \mathrm{mg} / \mathrm{kg}$ ) the number of newly formed cells decreased by about $50 \%[14]$. Our data show the stimulatory impact of olanzapine on adult neurogenesis in the adult SVZ, suggesting a modulatory effect of this drug after 4 weeks. Comparing brain slices from experimental and control groups, there were no significant differences in DCX-immunoreactive cells spatial localization and morphology. In another study, the neurogenic effect of olanzapine was marginal and limited to the prefrontal cortex (PFC) and dorsal striatum [15]. Moreover, the newborn cells in the PFC did not show the NeuN expression. It should be noted that in this 
experiment the antipsychotics were administered for a shorter period (3 weeks) in comparison with our study. Conflictingly, after extended (28-days) clozapine administration, a strong increase in cell proliferation was observed in the dentate gyrus [16]. Comparing the pro-neurogenic effects of haloperidol with other neuroleptics, it has to be stated, that risperidone, clozapine and olanzapine have a higher neurogenic potential than haloperidol [15]. The proneurogenic effect of haloperidol is caused by dopamine $D_{2}$ receptors inhibition. NSCs in the adult rat brain express $D_{2}$ receptors in the cytoplasmic membrane and their stimulation suppresses cell proliferation[3]. Haloperidol effects on the $D_{2}$ receptor antagonist prevent the negative effect of dopamine on NSCs mitotic potential [17]. Haloperidol can also increase the level of prolactin which has proneurogenic properties and increases neurogenesis in pregnant mice[18]. Prolactin may act directly by its receptors located on NSCs and indirectly by increasing the expression of the anti-apoptotic Bcl-2 protein [19]. Moreover, haloperidol increases expression of the matrix metalloproteinases and nerve growth factor (NGF) which influences the proliferation and survival of NSCs [20]. It should be emphasized that haloperidol has certain cytotoxic properties and it may cause neuronal damage via its cationic metabolites (HPP+, RHPP+) that are transported to several brain structures including SVZ leading to neurodegeneration. Haloperidol also increases metabolism of dopamine, decreases glutathione content, and induces NF-kB transcription factor, as it has been stated in various studies (Kumari et al. 2017, Górska et al. 2015, Raudenska et al. 2013).

The effect of olanzapine was also investigated on a mouse cell line CF-1[21]. For this, they examined NSCs from SVZ-derived neurospheres after administration of olanzapine at three doses of $10 \mathrm{nM}, 100 \mathrm{nM}$ and $1 \mu \mathrm{M}$ respectively over 7 days. Cytological analysis using 5Bromo-2'-deoxyuridine (BrdU) revealed the greatest increase in neurogenesis at 10nM. The evaluation of aminergic receptor and protein expressions showed a modestly elevated serotonin 5-HT2A receptor and GFAP levels at all drug concentrations, however an increase in B-tubulin gene expression happened only at a dose of 10nM while in the case of GABA only at a dose of $1 \mu \mathrm{M}$ compared to the control sample. Relatively high doses of olanzapine also induced an increase in neuregulin-1 (Nrg-1) gene expression [21]. This report seems to be especially interesting because the alterations of neuregulin-1 signaling pathways have been reported in schizophrenia (Mostaid et al. 2016,Wang et al. 2015, Luo et al. 2014, Pitcher et al. 2011). Nevertheless, olanzapine did not affect expression of any other schizophrenia vulnerability genes. Importantly, the concentrations used in this in vitro 
study cannot be roughly compared with clinical dose data of olanzapine in patients with schizophrenia, because the initial doses are 10-100 times greater than that predicted to remain in human plasma after 75 hours while the receptor affinities are around 1-100 nM.

Here, we also analyzed the expression of Sox-2, an early marker of pluripotent stem cells, thus devoid of CNS specificity [22]. Our results show almost a double increase in the average number of anti-Sox2 antibody-labeled cells in the drug-exposed group. We additionally compared the immune-detection of Sox-2 by fluorescent staining, which additionally confirmed the effect of the drug on the number of Sox2-positive cells. These results may also be in line with a recent study showing that olanzapine and aripiprazole may promote or restore disturbances in the oligodendrocyte/GABAergic neuron differentiation functions of SVZ-derived progenitor cells (with proteoglycan NG2 expression) induced by the treatment with NMDA-R antagonist - MK801 (Kaneta et al. 2017). Similarly, another finding suggests that olanzapine but not haloperidol may abolish MK-801-induced cognitive impairments in mice via stimulating cell proliferation in the SGZ (Song et al. 2016). On the other hand, it was also reported that olanzapine at $100 \mu \mathrm{M}$ dose caused structural mitochondrial damage and ROS-dependent depolarization in human neuroblastoma $\mathrm{SH}$ SY5Y cells. The same study, however, shows the triggering of autophagy both in cell culture and in the mouse brain in vivo (at dose $2 \mathrm{mg} / \mathrm{kg}$ ), which protects them from potential antipsychotic neurotoxicity. Of note, the drug treatment did not markedly affect the viability of SH-SY5Y cells (Vucicevic et al. 2014).

In a recent study, reported the influence of a series of atypical neuroleptics on human induced pluripotent stem cell (hiPSC)-derived neural stem cell (NSC) differentiation[23]. These cells showed a significant increase in the expression of the early neuron markers, Sox-2 and Pax6 upon exposure to clozapine and risperidone, which corresponds to the results, stated earlier, for olanzapine. Another marker of neurogenesis was DCX, which characterizes neuroblasts in a highly selective manner. The results show a significant increase in the average number of DCX positive cells. Additionally, the outcomes in this study are similar to those published by Rojczyk et al., who used an identical dosage regimen and observed a significant increase in the expression of DCX protein within the hypothalamus[24]. They are also in line with our previous results showing that long-term (28-days) treatment with haloperidol increased the origin of DCX-expressing cells in the rat SVZ and SGZ [25]. Here, we show the effect of olanzapine at the early stages of the neurogenesis process, although it is difficult to ascertain what the molecular mechanism of 
these changes are, as not all NSCs express dopamine receptors - a proper target for inhibitory action of neuroleptics. It can, therefore, be assumed that they may activate other signaling pathways of these cells, such as Akt-mTORC1, TGF-B or neuregulin 1. In the next step, it will be necesary to investigate potential mechanims of olanzapine on adult neurogenesis in the SVZ.

Despite the limitations of our study, including low animal numbers, and the lack of Sox2/DCX gene expression;Our findings suggest that chronic administration of olanzapine can support adult neurogenesis in the adult SVZ and may facilitate the origin of the rostral migratory stream in rats. Although not fully translational to the human brain, our conclusion may encourage the further study of olanzapine neuropharmacology in the context of adult neurogenesis modulation. In the future, it would also be of interest to study detailed features and fate of newly formed neurons both in the SVZ and the SGZ; after treatment with classical and atypical antipsychotic drugs.

\section{CONCLUSIONS}

Neuronal stem cells with SOX-2 expression are most sensitive to long-term effects of olanzapine within the SVZ. Olanzapine may be ranked as an early-neurogenesis simulating factor within the SVZ. Chronic exposure to olanzapine may stimulate differentiation of cells within the SVZ into a DCX-positive state.

\section{FUNDING SUPPORT}

This work was supported by the Medical University of Silesia grant for Department of Histology KNW-1-064/K/7//.

\section{CONFLICT OF INTEREST STATEMENT}

The authors declare that the research was conducted in the absence of any commercial or financial relationships that could be construed as a potential conflict of interest. 


\section{REFERENCES}

[1] Fernandes C, Rocha NB, Rocha S, Herrera-Solis A, Salas-Pacheco J, Garcia-Garcia $F$, et al. Detrimental role of prolonged sleep deprivation on adult neurogenesis. Front Cell Neurosci. 2015;9:140.

[2] Yuan TF, Li J, Ding F, Arias-Carrion O. Evidence of adult neurogenesis in nonhuman primates and human. Cell Tissue Res 2014; 358:17-23.

[3] Hoglinger GU, Arias-Carrion O, Ipach B, Oertel WH. Origin of the dopaminergic innervation of adult neurogenic areas. J Comp Neurol 2014;522:2336-48.

[4] Alvarez-Buylla A, Lim DA. For the long run: maintaining germinal niches in the adult brain. Neuron 2004;41:683-6.

[5] Yuan TF, Arias-Carrion O. Adult neurogenesis in the hypothalamus: evidence, functions, and implications. CNS Neurol Disord Drug Targets 2011;10:433-9.

[6] Arias-Carrion O, Freundlieb N, Oertel WH, Hoglinger GU. Adult neurogenesis and Parkinson's disease. CNS Neurol Disord Drug Targets. 2007 Oct;6(5):326-35.

[7] Hoglinger GU, Alvarez-Fischer D, Arias-Carrion O, Djufri M, Windolph A, Keber U, et al. A new dopaminergic nigro-olfactory projection. Acta Neuropathol 2015;130:333-48.

[8] Bishara D, Olofinjana O, Sparshatt A, Kapur S, Taylor D, Patel MX. Olanzapine: a systematic review and meta-regression of the relationships between dose, plasma concentration, receptor occupancy, and response. J Clin Psychopharmacol 2013;33:32935.

[9] Olijslagers JE, Werkman TR, McCreary AC, Kruse CG, Wadman WJ. Modulation of midbrain dopamine neurotransmission by serotonin, a versatile interaction between neurotransmitters and significance for antipsychotic drug action. Curr Neuropharmacol 2006;4:59-68.

[10] Duggan L, Fenton M, Rathbone J, Dardennes R, El-Dosoky A, Indran S. Olanzapine for schizophrenia. Cochrane Database Syst Rev 2005; 18:CD001359.

[11] Wakade CG, Mahadik SP, Waller JL, Chiu FC. Atypical neuroleptics stimulate neurogenesis in adult rat brain. J Neurosci Res 2002;69:72-9.

[12] Chikama K, Yamada H, Tsukamoto T, Kajitani K, Nakabeppu Y, Uchimura N. Chronic atypical antipsychotics, but not haloperidol, increase neurogenesis in the hippocampus of adult mouse. Brain Res 2017;1676:77-82.

[13] Nandra KS, Agius M. The differences between typical and atypical antipsychotics:

the effects on neurogenesis. Psychiatr Danub 2012;24 Suppl 1:S95-9.

[14] Backhouse B, Barochovsky O, Malik C, Patel AJ, Lewis PD. Effects of haloperidol on cell proliferation in the early postnatal rat brain. Neuropathol Appl Neurobiol. 1982;8:109-16. [15] Wang HD, Dunnavant FD, Jarman T, Deutch AY. Effects of antipsychotic drugs on neurogenesis in the forebrain of the adult rat. Neuropsychopharmacology 2004;29:1230-8. [16] Halim ND, Weickert CS, McClintock BW, Weinberger DR, Lipska BK. Effects of chronic haloperidol and clozapine treatment on neurogenesis in the adult rat hippocampus. Neuropsychopharmacology. 2004;29:1063-9. 
[17] Kippin TE, Kapur S, van der Kooy D. Dopamine specifically inhibits forebrain neural stem cell proliferation, suggesting a novel effect of antipsychotic drugs. J Neurosci 2005; ;25:5815-23.

[18] Shingo T, Gregg C, Enwere E, Fujikawa H, Hassam R, Geary C, et al. Pregnancystimulated neurogenesis in the adult female forebrain mediated by prolactin. Science 2003;299:117-20.

[19] Torner L, Karg S, Blume A, Kandasamy M, Kuhn HG, Winkler J, et al. Prolactin prevents chronic stress-induced decrease of adult hippocampal neurogenesis and promotes neuronal fate. J Neurosci 2009;29:1826-33.

[20] Keilhoff G, Grecksch G, Bernstein HG, Roskoden T, Becker A. Risperidone and haloperidol promote survival of stem cells in the rat hippocampus. Eur Arch Psychiatry Clin Neurosci 2010;260: 151-62.

[21] Councill JH, Tucker ES, Haskell GT, Maynard TM, Meechan DW, Hamer RM, et al. Limited influence of olanzapine on adult forebrain neural precursors in vitro. Neuroscience 2006;140:111-22.

[22] Baer K, Eriksson PS, Faull RL, Rees MI, Curtis MA. Sox-2 is expressed by glial and progenitor cells and Pax-6 is expressed by neuroblasts in the human subventricular zone. Exp Neurol 2007;204:828-31.

[23] Asada M, Mizutani S, Takagi M, Suzuki H. Antipsychotics promote neural differentiation of human iPS cell-derived neural stem cells. Biochem Biophys Res Commun 2016;480:615-21.

[24] Rojczyk E, Palasz A, Wiaderkiewicz R. Effects of neuroleptics administration on adult neurogenesis in the rat hypothalamus. Pharmacol Rep 2015;67:1208-14.

[25] Palasz A, Respondek K, Rojczyk E, Bogus K, Filipczyk L, Krzystanek M, Wiaderkiewicz R. The long term administration of haloperidol supports the origin of DCXexpressing cells in the adult rat brain. Farmakoter Psychiatr Neurol 2016; 32: 79-84.

Figure captions

Fig. (1). Expressions of Sox-2 and doublecortin (DCX) in the rat SVZ. Immunohistochemical DAB staining for Sox-2; control (A), olanzapine (B) and DCX; control (C), olanzapine (D). Scale bars; $100 \mu \mathrm{m}$. Number of Sox-2 (E) and DCX (F) immunopositive cells $(\mathrm{n} / \mathrm{mm})$ in the SVZ, ${ }^{* * *} p<0.05$.

Fig. (2). Immunofluorescence histochemistry for Sox-2 in the rat SVZ; control (FITC;A, FITC+DAPI; B), olanzapine (FITC;C, FITC+DAPI; D). Scale bars; $100 \mu \mathrm{m}$. 